\title{
Assessment of cardiac inflammation and remodeling during the development of streptozotocin-induced diabetic cardiomyopathy in vivo: A time course analysis
}

\author{
P.M. BECHER ${ }^{1}$, D. LINDNER ${ }^{1}$, M. FRÖHLICH ${ }^{1}$, K. SAVVATIS ${ }^{1,2}$, \\ D. WESTERMANN ${ }^{1}$ and C. TSCHÖPE ${ }^{1-3}$
}

\begin{abstract}
${ }^{1}$ Department of Cardiology and Pneumology, Charité University Hospital Berlin, Campus Benjamin Franklin, D-12200 Berlin; ${ }^{2}$ Berlin-Brandenburg Center for Regenerative Therapies, Charité University Hospital Berlin, Campus Virchow, D-13353 Berlin; ${ }^{3}$ German Centre for Cardiovascular Research (DZHK), Partner Site Berlin, Charité, D-13347 Berlin, Germany
\end{abstract}

Received February 28, 2013; Accepted April 23, 2013

DOI: $10.3892 /$ ijmm.2013.1368

\begin{abstract}
In this study, we examined cardiac inflammation, fibrosis and left ventricular (LV) function during the development of streptozotocin (STZ)-induced diabetic cardiomyopathy using an animal model of diabetes mellitus (DM). Diabetes was induced in 22 Sprague-Dawley rats by an intraperitoneal single injection of STZ $(70 \mathrm{mg} / \mathrm{kg})$. Non-diabetic animals served as the controls $(n=6)$. LV function was documented using the conductance catheter technique 2 and 6 weeks after the induction of diabetes. Cardiac tissue was analyzed for cardiac immune cell infiltration, oxidative stress and remodeling in rats with STZ-induced diabetes at 2 different time points by immunohistochemistry. Cardiac function was significantly impaired in the diabetic animals. After 2 weeks, the induction of diabetes resulted in impaired cardiac function indexed by a decrease in systolic and diastolic LV function. This impairment of LV performance continued for up to 6 weeks after the STZ injection. This was associated with an increase in cardiac $\mathrm{CD}^{+}$and $\mathrm{CD}^{+} \mathrm{a}^{+}$immune cell invasion and fibrosis, indexed by an increase in collagen content $(\mathrm{p}<0.05)$. Furthermore, oxidative stress response and matrix remodeling were increased after 2 weeks and this continued for up to 6 weeks after the induction of diabetes. In conclusion, cardiac dysfunction is associated with cardiac inflammation and adverse remodeling in experimental diabetic cardiomyopathy. Our results suggest that the model of STZ-induced diabetic cardiomyopathy is a robust model for investigating cardiac immune response and LV remodeling processes under diabetic conditions.
\end{abstract}

Correspondence to: Dr Carsten Tschöpe, Department of Cardiology and Pneumology, Charité University Hospital Berlin, Campus Benjamin Franklin, Hindenburgdamm 30, D-12200 Berlin, Germany E-mail: carsten.tschoepe@charite.de

Key words: diabetic cardiomyopathy, remodeling, inflammation, cardiac function

\section{Introduction}

Diabetes mellitus (DM) is one of the most important risk factors for heart failure and increased morbidity and mortality (1). There is growing evidence that the increased risk of heart failure may occur independently of accelerated coronary artery disease and arterial hypertension, suggesting that other mechanisms associated with diabetes underlie the development of cardiomyopathy $(2,3)$.

In addition, a number of studies have demonstrated that hyperglycemia directly causes cardiac damage, contributing to the development of diabetic cardiomyopathy $(4,5)$. However, numerous pathophysiological stimuli are involved in its development and mediate cardiac injury, leading to systolic and diastolic left ventricular (LV) dysfunction (6). The pathophysiology of diabetic cardiomyopathy includes, for example, microangiopathy, endothelial dysfunction, cardiac fibrosis and the disruption of intracellular $\mathrm{Ca}^{2+}$ transport, all triggered by the diabetic milleu (7-9). Moreover, structural changes in extracellular matrix (ECM) regulation and the accumulation of cardiac fibrosis, an intensified production of oxidative stress and an overwhelming cardiac inflammatory immune response play a crucial role in the pathogenesis of diabetic cardiomyopathy $(6,7)$.

Previous studies have demonstrated the impaired LV performance under basal conditions during the chronic stage of streptozotocin (STZ)-induced diabetic cardiomyopathy. Furthermore, in previous studies, we identified possible pathophysiological mechanisms, resulting in the cardiac phenotype of diabetic cardiomyopathy. Among others, we found that cardiac fibrosis, endothelial dysfunction, cardiac inflammation, as well as neurohumoral activation are greatly involved in the development of cardiac dysfunction under those conditions (7,9-11).

The rat/mouse model of diabetic cardiomyopathy induced by an injection of STZ is a well-established model for investigating this condition. The cardiac phenotype under basal conditions in the chronic stage has been sufficiently characterized. Thus, in the current study, by time course analysis, 
we investigated LV performance by in vivo pressure-volume loops and adverse cardiac remodeling using a rat model of STZ-induced diabetic cardiomyopathy. We measured LV function, cardiac immune cell invasion, oxidative stress, as well as cardiac remodeling under diabetic conditions.

\section{Materials and methods}

Animal characteristics and study design. Six-week-old Sprague-Dawley rats $(\mathrm{n}=22)$ were maintained on a 12:12 h light-dark cycle and fed with standard chow ad libidum. In 12 rats, diabetes was induced by an intraperitoneal (i.p.) single injection of STZ $(70 \mathrm{mg} / \mathrm{kg})$ diluted in $0.1 \mathrm{M}$ sodium citrate buffer, pH 4.5 (Sigma-Aldrich Chemie Gmbh, Munich, Germany); the rats were then randomly divided into 2 experimental groups (STZ 2 weeks and STZ 6 weeks). Hyperglycemia was measured $48 \mathrm{~h}$ later using a reflectance meter (Acutrend; Roche Diagnostics GmbH, Mannheim, Germany). All diabetic animals displayed a blood glucose level $>550 \mathrm{mg} / \mathrm{dl}$ associated with severe polyuria and polydipsia. For the controls, 6 vehicletreated animals (treated with citrate buffer only) were used. All animal experiments were carried out in accordance with the Guide for the Care and Use of Laboratory Animals published by the US NIH (NIH Publication no. 85-23, revised 1996).

Surgical procedures and hemodynamic measurements. Two (STZ 2 weeks) and 6 (STZ 6 weeks) weeks after the STZ injection, the animals were anesthetized (pentobarbital $60 \mathrm{mg} / \mathrm{kg}$, i.p. injection; and buprenorphine $0.1 \mathrm{mg} / \mathrm{g}$, i.p. injection), intubated and artificially ventilated. A 2 French micro-conductance catheter (Aria SPR 858; Millar Instruments, Inc., Houston, TX, USA) was positioned in the LV for the continuous registration of LV pressure-volume (PV) loops in a closed-chest model. Calibration of the volume signal was obtained using the hypertonic saline (10\%) wash-in technique. Indices of cardiac function were derived from PV data obtained both at the basal steady state and during transient preload reduction by temporary occlusion of the abdominal vena cava. All hemodynamic measurements were performed during apnea. After the euthanization of the mice, LV tissues were excised, immediately snap-frozen in liquid nitrogen and stored at $-80^{\circ} \mathrm{C}$ for biological and immunohistological analyses.

Immunohistological measurements. The total collagen content of the Sirius red (Polysciences, Inc., Warrington, PA, USA)stained sections was measured under circularly polarised light as previously described (12). The data were then quantified by digital image analysis as the percentage of area fraction.

As previously described $(13,14)$, LV tissue of the left ventricle was embedded in Tissue-Tek (Dako) and immunohistochemistry was performed with specific antibodies directed against CD3 (Bioss Inc., Woburn, MA, USA), CD8a (Bioss Inc.), nitrotyrosin (Sigma-Aldrich Chemie Gmbh), $\alpha$-smooth muscle actin (SMA) (Abcam, Cambridge, UK) and matrix metalloproteinase (MMP)-2 (Chemicon, Temecula, CA, USA). Quantification was performed by digital image analyses $(13,14)$. In brief, the ratio between the heart tissue area and the specific chromogen-positive area was calculated (area fraction, \%). The numbers of infiltrating cells were calculated by measuring the number of cells/area of heart tissue $\left(\right.$ cells $\left./ \mathrm{mm}^{2}\right)$.
Statistical analysis. Statistical analysis was performed using SPSS version 12.0 software. Data are expressed as the means \pm SEM. Statistical differences were assessed using the Kruskal-Wallis test in conjunction with the Mann-Whitney U post hoc test. Bonferroni correction was applied to the post hoc Mann-Whitney $\mathrm{U}$ test to adjust for multiple comparisons. Pearson's correlation co-efficient was used for linear regression analysis and Spearman's correlation co-efficient was used for non-linear correlations. Regression analyses and curve fitting were performed to determine exact correlations. Differences were considered statistically significantly at a value of $\mathrm{p}<0.05$.

\section{Results}

Animal characteristics and hemodynamic measurements after the induction of diabetes. Two and 6 weeks after STZ-induced type I diabetes, animal characteristics, systolic and diastolic LV function were determined in all experimental groups using the conductance catheter technique (Table I).

At the early time point of 2 weeks, a significant decrease $(-33 \%)$ in heart rate was observed in the diabetic animals. At the later time point of 6 weeks, no change in heart rate was observed (Fig. 1A). This was associated with a reduction in diastolic LV function indicated by a significant reduction of LV relaxation at the early time point $(-42 \%)$. Six weeks after STZ-induced diabetes, an additional decrease in LV relaxation $(-38 \%)$ was observed (Fig. 1D). In addition, STZ-induced diabetes resulted in a significant increase $(+38 \%)$ in cardiac stiffness at the later time point (Fig. 1E). Systolic LV function, indexed by LV contractility, displayed a significant reduction of $-22 \%$ at the early time point of the disease, leading to an additional decrease of another $-26 \%$ after 6 weeks (Fig. 1B); the end systolic pressure exhibited the same pattern (Fig. 1C). The initial decrease $(-18 \%)$ was followed by a second significant $(-26 \%)$ decrease, indicating global LV dysfunction. All these results were associated with a significant reduction $(-47 \%)$ in cardiac output after 2 and 6 weeks (Fig. 1F).

ECM alterations and remodeling after the induction of diabetes. To examine the turn over of the ECM assembly, cardiac tissue was stained by Sirius red measuring the total content of collagen in the cardiac tissue. Moreover, $\alpha$-SMA and MMP-2 protein expression levels were measured by specific staining. The diabetic animals showed an initial increase (1.9-fold) in total collagen, but there was no additional increase at the later time point (Fig. 2). By contrast, the investigation of $\alpha$-SMA and MMP-2 protein expression levels revealed an initial 16.6-fold $(\mathrm{p}<0.05)$ and 11.6-fold $(\mathrm{p}<0.05)$ increase after 2 weeks, respectively. Six weeks after the induction of diabetes by STZ, we observed an additional 1.6-fold $(\mathrm{p}<0.05)$ and 5-fold $(\mathrm{p}>0.05)$ increase in $\alpha$-SMA and MMP-2 protein expression levels in the cardiac tissue, respectively (Fig. 3C and D).

Cardiac immune cell infiltration and oxidative stress response after the induction of diabetes. To investigate the immune cell infiltration by $\mathrm{T}$ cells, we measured the number of $\mathrm{CD}^{+}$ and $\mathrm{CD}^{+} \mathrm{a}^{+}$immune cells in the cardiac tissue by specific immunological staining, as well as the content of nitrotyrosin, to evaluate oxidative stress response in the cardiac tissue. The number of $\mathrm{CD}^{+}$immune cells significantly increased 
Table I. Animal characteristics and hemodynamic results 2 and 6 weeks after the induction of diabetes.

\begin{tabular}{|c|c|c|c|}
\hline Items & Control & STZ 2 weeks & STZ 6 weeks \\
\hline \multicolumn{4}{|l|}{ Characteristics } \\
\hline Body weight (g) & $491 \pm 10$ & $374 \pm 9^{a}$ & $253 \pm 7^{a}$ \\
\hline Blood glucose (mmol/l) & $4.1 \pm 0.3$ & $>30.5$ & $>30.5$ \\
\hline Mean blood pressure & $113 \pm 7$ & $94 \pm 8^{a}$ & $73 \pm 9^{\mathrm{a}, \mathrm{b}}$ \\
\hline Heart weight (mg) & $1223 \pm 16$ & $1009 \pm 19^{a}$ & $839 \pm 21^{\mathrm{a}}$ \\
\hline LV weight (mg) & $883 \pm 20$ & $697 \pm 16^{\mathrm{a}}$ & $586 \pm 18^{a}$ \\
\hline Heart weight/body weight $\times 10^{-3}$ & $2.49 \pm 0.06$ & $2.67 \pm 0.09^{\mathrm{a}}$ & $3.31 \pm 0.07^{\mathrm{a}}$ \\
\hline LV weight/heart weight & $0.726 \pm 0.02$ & $0.68 \pm 0.03^{\mathrm{a}}$ & $0.69 \pm 0.02^{\mathrm{a}}$ \\
\hline \multicolumn{4}{|l|}{ Global LV function } \\
\hline Heart rate (bpm) & $378 \pm 13$ & $257 \pm 10^{\mathrm{a}}$ & $229 \pm 9^{a}$ \\
\hline LV end systolic pressure (mmHg) & $116 \pm 4$ & $97.4 \pm 6^{\mathrm{a}}$ & $76 \pm 7^{\mathrm{a}, \mathrm{b}}$ \\
\hline Cardiac output ( $\mu 1 / \mathrm{min})$ & $95872 \pm 10723$ & $50893 \pm 8983^{\mathrm{a}}$ & $53211 \pm 5213^{\mathrm{a}}$ \\
\hline Ejection fraction $(\%)$ & $52.7 \pm 4.8$ & $45.2 \pm 5.5$ & $49 \pm 3.9$ \\
\hline \multicolumn{4}{|l|}{ Systolic LV function } \\
\hline LV end systolic volume $(\mu \mathrm{l})$ & $256 \pm 42$ & $272 \pm 52$ & $268 \pm 32$ \\
\hline LV contractility (mmHg/sec) & $7234 \pm 365$ & $5671 \pm 331^{\mathrm{a}}$ & $4178 \pm 245^{\mathrm{a}, \mathrm{b}}$ \\
\hline LV end systolic pressure (mmHg) & $115.3 \pm 3.9$ & $94.8 \pm 4.2^{\mathrm{a}}$ & $76 \pm 6.1^{\mathrm{a}, \mathrm{b}}$ \\
\hline \multicolumn{4}{|l|}{ Diastolic LV function } \\
\hline LV end diastolic volume $(\mu \mathrm{l})$ & $489 \pm 46$ & $446 \pm 61$ & $477 \pm 51$ \\
\hline $\mathrm{LV}$ diastolic relaxation $(\mathrm{mmHg} / \mathrm{sec})$ & $-6598 \pm 195$ & $-3723 \pm 201^{a}$ & $-2874 \pm 202^{a, b}$ \\
\hline LV diastolic relaxation time (msec) & $13.6 \pm 0.8$ & $21.4 \pm 0.7^{\mathrm{a}}$ & $23.5 \pm 0.9^{\mathrm{a}}$ \\
\hline LV end diastolic pressure (mmHg) & $4.1 \pm 1$ & $5.3 \pm 0.8$ & $6.1 \pm 1$ \\
\hline LV pressure half time (msec) & $8.2 \pm 0.3$ & $12.8 \pm 0.7^{\mathrm{a}}$ & $14.2 \pm 1.1^{\mathrm{a}}$ \\
\hline Stiffness constant $\beta$ & $0.0054 \pm 0.001$ & $0.0086 \pm 0.003$ & $0.0142 \pm 0.003^{\mathrm{a}}$ \\
\hline
\end{tabular}

a $<<0.05$ vs. control; ${ }^{\mathrm{b}} \mathrm{p}<0.05$ vs. control and STZ 2 weeks group. STZ, streptozotocin; LV, left ventricular.
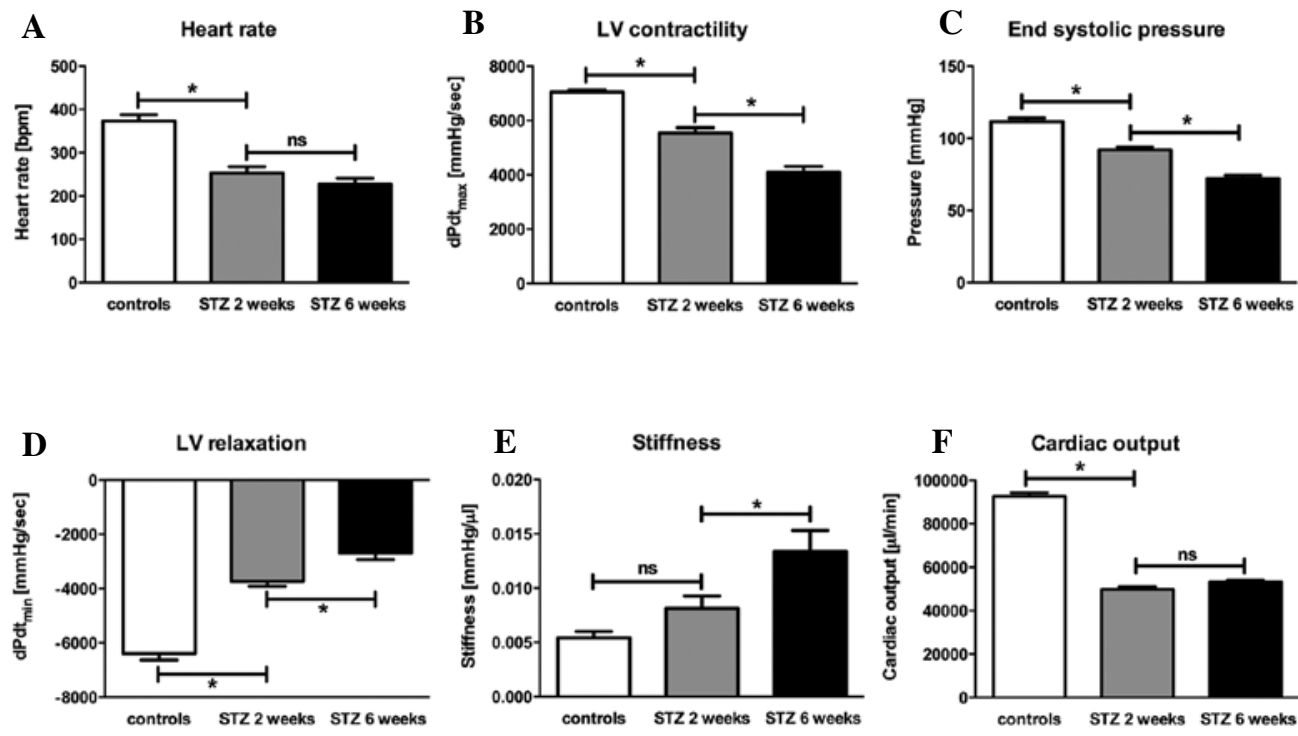

Figure 1. Heart rate, left ventricular (LV) contractility, end systolic pressure, LV relaxation, cardiac stiffness and cardiac output as representative hemodynamic parameters of control rats and rats with streptozotocin (STZ)-induced diabetes in a time course analysis. Data are from 6-8 rats/group. Data are expressed as the means \pm SEM. " $\mathrm{p}<0.05$.

at 2 and 6 weeks after the induction of diabetes (Fig. 3A; $\mathrm{p}<0.05)$. Of note, the number of $\mathrm{CD}^{+}$cells in the diabetic mice displayed an initial 3.6-fold $(\mathrm{p}<0.05)$ increase followed by a significant decrease after 6 weeks (Fig. 3B). By contrast, the level of nitrotyrosin showed a constant 7.8-fold $(\mathrm{p}<0.05)$ increase followed by another 1.4 -fold $(\mathrm{p}<0.05)$ increase in the animals with STZ-induced diabetes compared with the controls (Fig. 3E). 


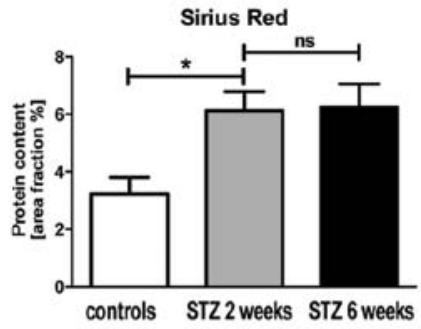

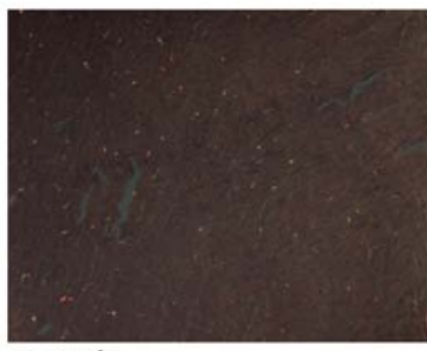

controls

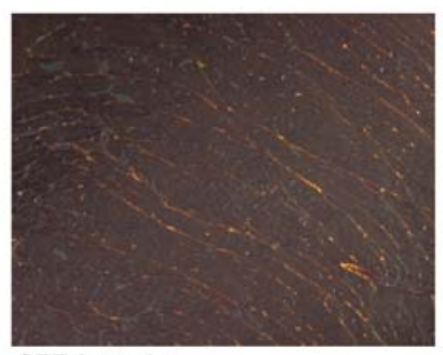

STZ 2 weeks

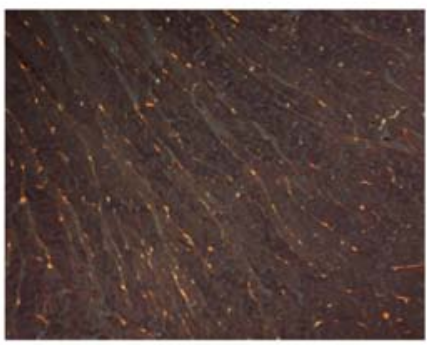

STZ 6 weeks

Figure 2. Representative images of Sirius red staining in the cardiac tissue of the control rats and rats with streptozotocin (STZ)-induced diabetes. Data are from 6-8 rats/group. Data are expressed as the means \pm SEM. ${ }^{*} \mathrm{p}<0.05$.

A

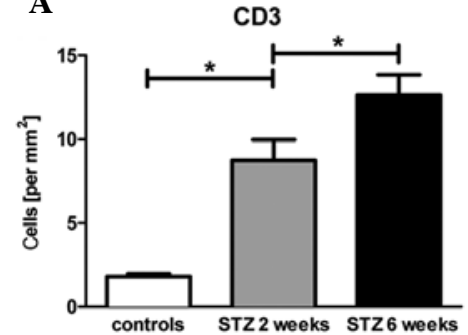

B

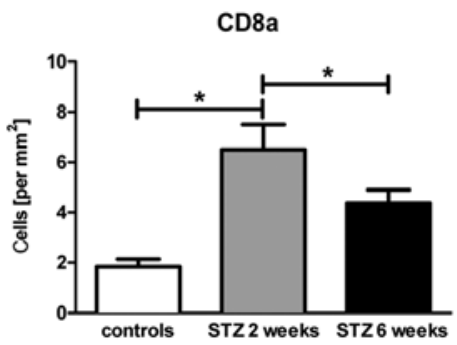

C

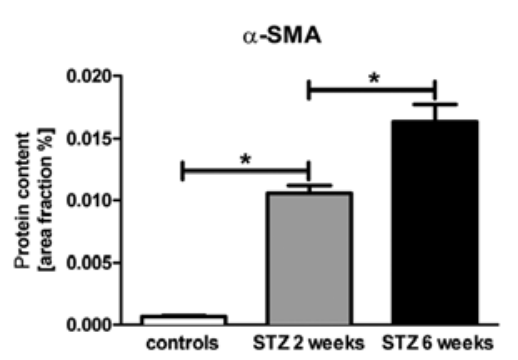

D

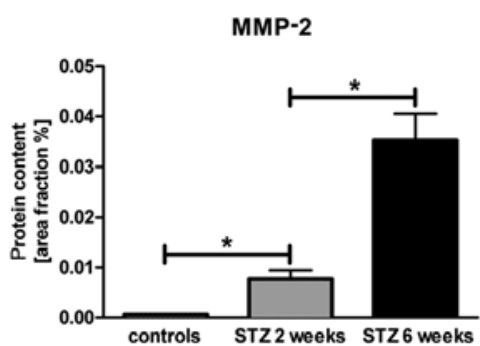

$\mathbf{E}$

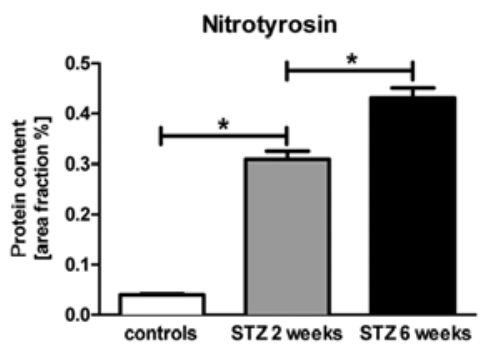

Figure 3. Cardiac immune cell infiltration of $\mathrm{CD}^{+}$and $\mathrm{CD} 8 \mathrm{a}^{+} \mathrm{T}$ lymphocytes and protein expression levels of nitrotyrosin, $\alpha$-SMA and MMP-2 in the cardiac tissue of the control rats and rats with streptozotocin (STZ)-induced diabetes. Data are from 6-8 rats/group. Data are expressed as the means \pm SEM. "p $<0.05$.

Correlations between hemodynamic and morphological parameters after the induction of diabetes. We examined the possible correlations between hemodynamic and morphological parameters in this experimental setting. We investigated the correlation between the total content of collagen and systolic LV function, indexed by LV contractility and end systolic pressure. We found that an increase in total collagen content correlated with a significant reduction in systolic LV function $(\mathrm{r}=0.55, \mathrm{r}=0.6$; Fig. 4A and B). Concerning the diastolic LV function, we investigated the pathophysiological correlation between the protein content of $\alpha$-SMA and LV stiffness. We determined a correlation between the increased number of
$\alpha$-SMA-positive myofibroblasts and the increase in cardiac stiffness ( $\mathrm{r}=0.7$; Fig. 4C).

Moreover, we performed correlation analyses to determine the correlation between cardiac immune cell infiltration and the parameters of systolic and diastolic LV function. We found that an increased cardiac $\mathrm{CD}^{+}$immune cell infiltration correlated with a reduction in $\mathrm{LV}$ contractility $(\mathrm{r}=0.83)$, end systolic pressure ( $\mathrm{r}=0.77)$ and an increase in cardiac stiffness ( $\mathrm{r}=0.54)$ (Fig. 5A-C). In addition, we also investigated possible correlations between the number of $\mathrm{CD} 8 \mathrm{a}^{+}$immune cells and these hemodynamic parameters. It was also found that an increased number of $\mathrm{CD} \mathrm{a}^{+}$immune cells correlated with a reduction in 

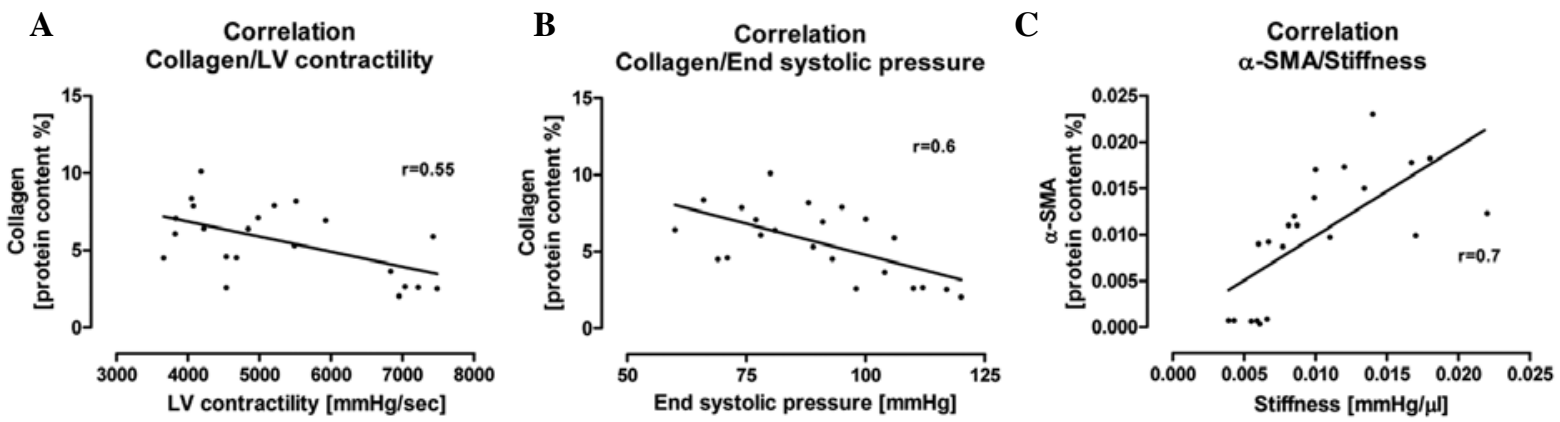

Figure 4. Correlations between protein expression levels of collagen content/ left ventricular (LV) contractility, collagen content/end systolic pressure, as well as protein expression levels of $\alpha$-SMA/cardiac stiffness in the control rats and rats with streptozotocin (STZ)-induced diabetes. Data are from 6-8 rats/group.

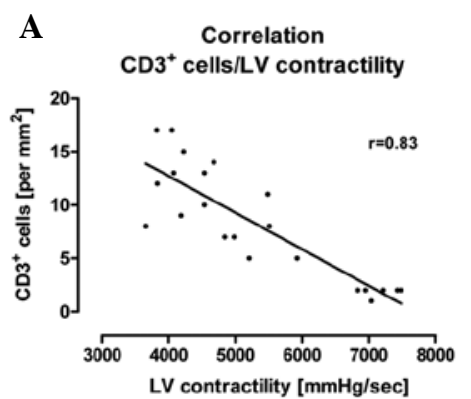

D

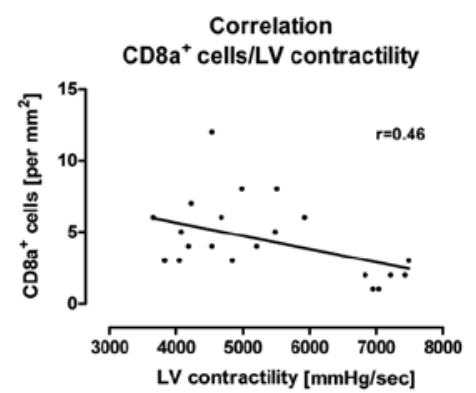

B

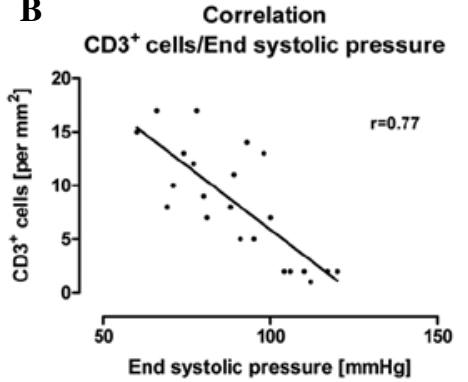

$\mathbf{E}$

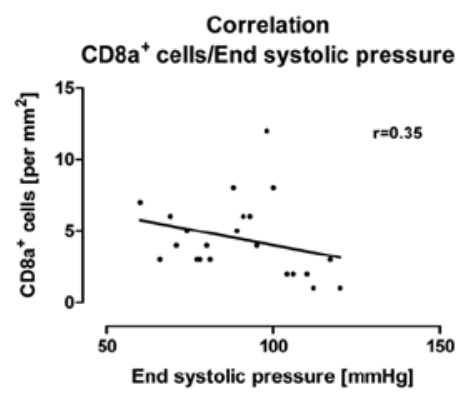

C

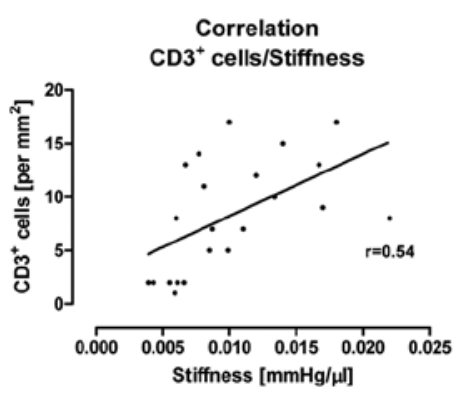

$\mathbf{F}$

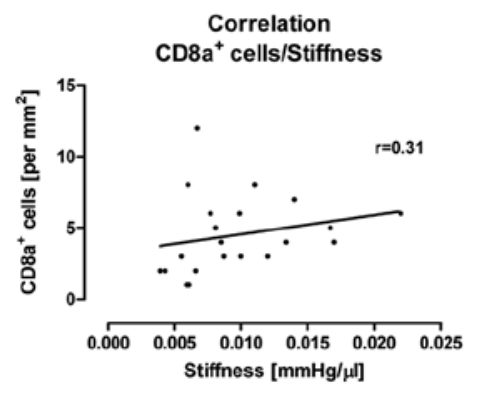

Figure 5. Correlations between $\mathrm{CD}^{+}$and $\mathrm{CD} 8 \mathrm{a}^{+}$immune cells and left ventricular (LV) contractility, end systolic pressure, as well as cardiac stiffness in control rats and rats with streptozotocin (STZ)-induced diabetes. Data are from 6-8 rats/group.

LV contractility $(\mathrm{r}=0.48)$, end systolic pressure $(\mathrm{r}=0.35)$ and an increase in cardiac stiffness ( $\mathrm{r}=0.31$ ) (Fig. 5D-F). However, the correlation between $\mathrm{CD} 8 \mathrm{a}^{+}$immune cell infiltration and hemodynamic parameters was weaker when compared with the number of infiltrating $\mathrm{CD}^{+}{ }^{+}$immune cells and the hemodynamic parameters (Fig. 5). Furthermore, we observed that a significant increase in $\mathrm{CD}^{+}$and $\mathrm{CD} \mathrm{a}^{+}$immune cells correlated with an increase in collagen and $\alpha$-SMA protein content in the cardiac tissue after the induction of diabetes (Fig. 6).

\section{Discussion}

The salient finding of this study is that the model of STZ-induced diabetic cardiomyopathy is a robust model for the investigation of cardiac inflammation and remodeling processes. Our data demonstrate that experimental diabetic cardiomyopathy is characterized by an increase in cardiac inflammation and changes in the regulation of the ECM over a time period of 6 weeks following STZ-induced-diabetes.
Diabetic cardiomyopathy is associated with LV dysfunction $(12,15)$. The results of the present study revealed an impairment in diastolic and systolic LV function at 2 and 6 weeks after the induction of diabetic cardiomyopathy. These hemodynamic results are in line with those from previous studies using the same experimental rat model $(7,9,11,16)$. However, we also described the concrete time course of the impairment in systolic and diastolic LV performance over a period of 2-6 weeks, indicated by a decrease in LV contractility, end systolic pressure, LV relaxation and an increase cardiac stiffness, all resulting in a significant reduction in cardiac output.

Our hemodynamic findings identified an early diastolic LV dysfunction, which showed a clear progression over time in this animal model. Active and passive diastolic LV relaxation was affected by the STZ injection. LV relaxation as a marker for active LV relaxation was significantly impaired 2 weeks after the induction of diabetes, whereas cardiac stiffness as a marker for passive LV relaxation displayed a tendency of deterioration after 2 weeks, but was only impaired 6 weeks after the induc- 
$\mathbf{A}$

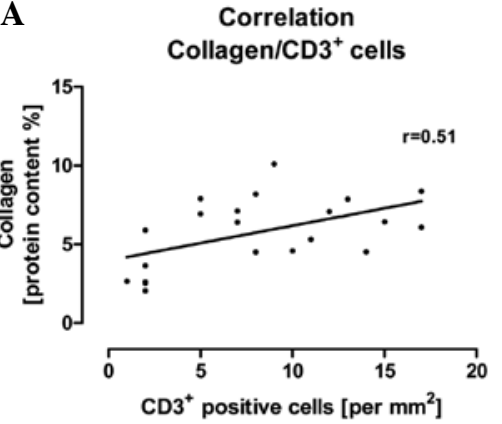

C

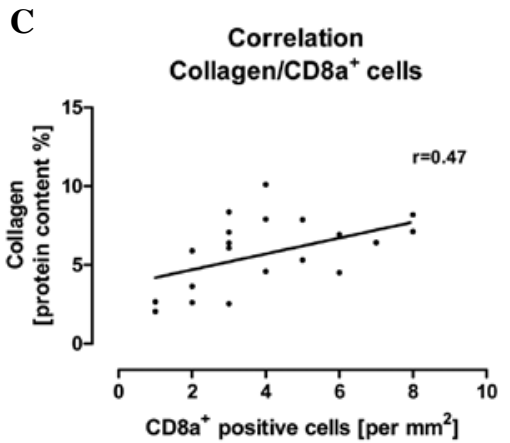

B

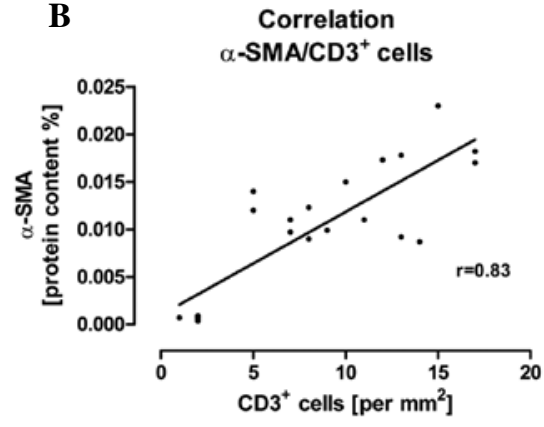

D

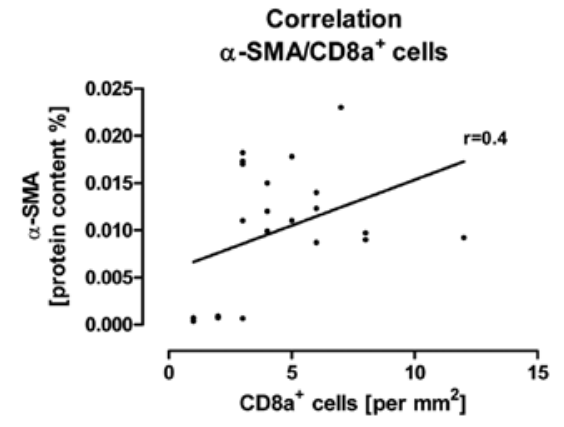

Figure 6. Correlations between $\mathrm{CD}^{+}$and $\mathrm{CD} 8 \mathrm{a}^{+}$immune cells and the protein content of collagen and $\alpha$-SMA in control rats and rats with streptozotocin (STZ)induced diabetes. Data are from 6-8 rats/group.

tion of diabetes. The end diastolic pressure was unaffected in this experimental setting; there was no significant increase in end systolic pressure following STZ-induced diabetes (Table I). This may be explained by chronic dehydration in the animals with STZ-induced diabetes, leading to a reduction in afterload. In previous studies, we demonstrated that an induction of cardiac inflammation under diabetic conditions was associated with an impairment in systolic LV function $(17,18)$. In the current study, we confirmed the hemodynamic profile of systolic and diastolic LV dysfunction under diabetic conditions.

Cardiac inflammation is one of the hallmarks of heart failure $(6,9)$. Intensified pro-inflammatory cytokine expression levels, as well as increased immune cell infiltration, such as cytotoxic T lymphocytes and macrophages, has been observed in the inflamed heart in diabetic cardiomyopathy (19-21). As regards this finding, we examined the invasion of $\mathrm{CD}^{+}$and $\mathrm{CD} 8 \mathrm{a}^{+}$ immune cells into the heart in this disease model. The induction of diabetes led to an uninterrupted increase in $\mathrm{CD}^{+}$immune cell invasion over the 6 -week observation period. In addition, the induction of diabetes by STZ led to a significant increase in the number of CD8 $\mathrm{a}^{+} \mathrm{T}$ lymphocytes 2 weeks after the STZ injection. Of note, 6 weeks after the induction of diabetes, the cardiac amount of this cell population was reduced when compared to the time point of 2 weeks after the STZ injection, indicating an important role of $\mathrm{CD} 8 \mathrm{a}^{+}$immune cells predominantly during the early stages of diabetic cardiomyopathy.

A large body of evidence indicates that LV remodeling accompanied with changes in ECM regulation is an important factor for LV function in diabetic cardiomyopathy $(11,16)$. In this study, the induction of diabetes led to a significant increase in cardiac fibrosis, indexed by an increase in total collagen content at the time points of 2 and 6 weeks after the induction of diabetes. Moreover, we matched the values of total collagen and LV contractility. We showed that the total collagen content correlated with a reduction in LV contractility and end systolic pressure, suggesting that the corrrelation between systolic LV function and myocardial fibrosis plays a pathophysiological role in this experimental setting.

In the clinical course of heart failure, MMPs are upregulated by intense cardiac inflammation and may contribute to cardiac remodeling under diabetic conditions $(22,23)$. We therefore analysed the protein expression levels of MMP-2 and found increased protein expression levels of MMP-2 in this experimental setting.

However, the persistence of an abnormally high number of myofibroblasts is a hallmark of fibrotic disease in other organs, as well as the heart $(24,25)$. In the current study, we identified that STZ-induced diabetes led to an increase in the number of $\alpha$-SMA-positive myofibroblasts in the heart 2 weeks after the STZ injection. However, an additional marked increase in the number of $\alpha$-SMA-positive myofibroblasts was documented at the time point of 6 weeks after the STZ injection. Concerning these findings, we also observed a correlation between the number of $\alpha$-SMA-positive myofibroblasts and cardiac stiffness, as a strong marker for diastolic LV dysfunction. Of note, an increase in the number of $\alpha$-SMA-positive myofibroblasts correlated with an increase in the cardiac stiffness index, suggesting a pathophysiological impact of myofibroblasts in adverse myocardial remodeling during diabetic conditions in rats. Moreover, we verified that an increased cardiac immune cell invasion of $\mathrm{CD}^{+}$and $\mathrm{CD} 8^{+}$cells correlated with an increased protein content of collagen and $\alpha$-SMA in the cardiac tissue at 2 and 6 weeks after the induction of diabetes. In addition, we also showed that an increased infiltration of these immune cell populations was significantly associated with an impairment in systolic and diastolic LV performance. These 
results indicate an important role of $\mathrm{CD}^{+}$and $\mathrm{CD}^{2} \mathrm{a}^{+}$immune cells in the development and progression of LV dysfunction and adverse cardiac remodeling under diabetic conditions.

DM is associated with an exponential increase in oxidative damage (26). Previous studies have demonstrated that nitrotyrosin, as a maker of oxidative stress, can participate in adverse remodeling, contributing to the development of heart failure $(27,28)$. In line with this finding, we observed that the STZ injection led to an increase in nitrotyrosin protein expression levels, suggesting an important role of oxidative stress in the pathogenesis of diabetic cardiomyopathy.

Previous studies have reported the effects of exogenous insulin therapy. It has also been shown that insulin treatment alone cannot normalize heart function under diabetic conditions (29-31). However, future studies should include a third experimental group with insulin treatment.

In conclusion, the current study displayed the cardiac phenotype of rats with STZ-induced diabetes rats in a time course analysis. The induction of diabetes by STZ led to an impairment in systolic and diastolic LV function, associated with an increase in immune cell invasion and adverse cardiac remodeling. This study reveals an important role of the maintenance of cardiac structure, by regulating the ECM assembly, in diabetic cardiomyopathy. We hope that these new findings of cardiac performance and remodeling will increase our understanding of the pathophysiology and development of diabetic cardiomyopathy.

\section{Acknowledgements}

We thank Kerstin Puhl, Georg Zingler and Nadine Orrin for their excellent technical assistance. This study was funded by FP7-Health-2010, MEDIA (261409).

\section{References}

1. Poornima IG, Parikh P and Shannon RP: Diabetic cardiomyopathy: the search for a unifying hypothesis. Circ Res 98: 596-605, 2006.

2. Grundy SM, Benjamin IJ, Burke GL, et al: Diabetes and cardiovascular disease: a statement for healthcare professionals from the American Heart Association. Circulation 100: 1134-1146, 1999.

3. Howard BV and Wylie-Rosett J: Sugar and cardiovascular disease: a statement for healthcare professionals from the Committee on Nutrition of the Council on Nutrition, Physical Activity, and Metabolism of the American Heart Association. Circulation 106: 523-527, 2002.

4. Devereux RB, Roman MJ, Paranicas M, et al: Impact of diabetes on cardiac structure and function: the strong heart study. Circulation 101: 2271-2276, 2000.

5. Cai L, Li W, Wang G, Guo L, Jiang Y and Kang YJ: Hyperglycemia-induced apoptosis in mouse myocardium: mitochondrial cytochrome C-mediated caspase-3 activation pathway. Diabetes 51: 1938-1948, 2002.

6. Heymans S, Hirsch E, Anker SD, et al: Inflammation as a therapeutic target in heart failure? a scientific statement from the Translational Research Committee of the Heart Failure Association of the European Society of Cardiology. Eur J Heart Fail 11: 119-129, 2009.

7. Tschope C, Walther T, Koniger J, et al: Prevention of cardiac fibrosis and left ventricular dysfunction in diabetic cardiomyopathy in rats by transgenic expression of the human tissue kallikrein gene. FASEB J 18: 828-835, 2004.

8. Tschope C, Spillmann F, Rehfeld U, et al: Improvement of defective sarcoplasmic reticulum $\mathrm{Ca}_{2}{ }^{+}$transport in diabetic heart of transgenic rats expressing the human kallikrein-1 gene. FASEB J 18: 1967-1969, 2004.
9. Westermann D, Rutschow S, Jager S, et al: Contributions of inflammation and cardiac matrix metalloproteinase activity to cardiac failure in diabetic cardiomyopathy: the role of angiotensin type 1 receptor antagonism. Diabetes 56: 641-646, 2007.

10. Riad A, Westermann D, Van Linthout S, et al: Enhancement of endothelial nitric oxide synthase production reverses vascular dysfunction and inflammation in the hindlimbs of a rat model of diabetes. Diabetologia 51: 2325-2332, 2008.

11. Westermann D, Van Linthout S, Dhayat S, et al: Cardioprotective and anti-inflammatory effects of interleukin converting enzyme inhibition in experimental diabetic cardiomyopathy. Diabetes 56: 1834-1841, 2007.

12. Westermann D, Rutschow S, Van Linthout S, et al: Inhibition of p38 mitogen-activated protein kinase attenuates left ventricular dysfunction by mediating pro-inflammatory cardiac cytokine levels in a mouse model of diabetes mellitus. Diabetologia 49: 2507-2513, 2006.

13. Westermann D, Becher PM, Lindner D, et al: Selective PDE5A inhibition with sildenafil rescues left ventricular dysfunction, inflammatory immune response and cardiac remodeling in angiotensin II-induced heart failure in vivo. Basic Res Cardiol 107: 308, 2012.

14. Becher PM, Lindner D, Miteva K, et al: Role of heart rate reduction in the prevention of experimental heart failure: comparison between If-channel blockade and beta-receptor blockade. Hypertension 59: 949-957, 2012.

15. Wang J, Song Y, Elsherif L, et al: Cardiac metallothionein induction plays the major role in the prevention of diabetic cardiomyopathy by zinc supplementation. Circulation 113: 544-554, 2006.

16. Westermann D, Van Linthout S, Dhayat S, et al: Tumor necrosis factor-alpha antagonism protects from myocardial inflammation and fibrosis in experimental diabetic cardiomyopathy. Basic Res Cardiol 102: 500-507, 2007.

17. Van Linthout S, Riad A, Dhayat N, et al: Anti-inflammatory effects of atorvastatin improve left ventricular function in experimental diabetic cardiomyopathy. Diabetologia 50: 1977-1986, 2007.

18. Dorenkamp M,Riad A, Stiehl S, et al: Protection against oxidative stress in diabetic rats: role of angiotensin AT(1) receptor and beta 1-adrenoceptor antagonism. Eur J Pharmacol 520: 179-187, 2005.

19. Savvatis K, Westermann D, Schultheiss HP and Tschope C: Kinins in cardiac inflammation and regeneration: insights from ischemic and diabetic cardiomyopathy. Neuropeptides 44: $119-125,2010$.

20. Yeghiazaryan K, Bauriedel G, Schild HH and Golubnitschaja O: Prediction of degeneration of native and bioprosthetic aortic valves: issue-related particularities of diabetes mellitus. Infect Disord Drug Targets 8: 88-99, 2008.

21. Marwick TH: Diabetic heart disease. Heart 92: 296-300, 2006.

22. Tyagi SC and Hayden MR: Role of nitric oxide in matrix remodeling in diabetes and heart failure. Heart Fail Rev 8: 23-28, 2003.

23. Tsioufis C, Bafakis I, Kasiakogias A and Stefanadis C: The role of matrix metalloproteinases in diabetes mellitus. Curr Top Med Chem 2: 1159-1165, 2012.

24. Leask A: Potential therapeutic targets for cardiac fibrosis: TGFbeta, angiotensin, endothelin, CCN2, and PDGF, partners in fibroblast activation. Circ Res 106: 1675-1680, 2010.

25. Hammoud L, Lu X, Lei M and Feng Q: Deficiency in TIMP-3 increases cardiac rupture and mortality post-myocardial infarction via EGFR signaling: beneficial effects of cetuximab. Basic Res Cardiol 106: 459-471, 2011.

26. Bugger H and Abel ED: Mitochondria in the diabetic heart. Cardiovasc Res 88: 229-240, 2010.

27. Henderson BC and Tyagi SC: Oxidative mechanism and homeostasis of proteinase/antiproteinase in congestive heart failure. J Mol Cell Cardiol 41: 959-962, 2006.

28. La Rocca G, Di Stefano A, Eleuteri E, et al: Oxidative stress induces myeloperoxidase expression in endocardial endothelial cells from patients with chronic heart failure. Basic Res Cardiol 104: 307-320, 2009

29. Regan TJ, Wu CF, Yeh CK, Oldewurtel HA and Haider B: Myocardial composition and function in diabetes. The effects of chronic insulin use. Circ Res 49: 1268-1277, 1981.

30. Iribarren C, Karter AJ, Go AS, et al: Glycemic control and heart failure among adult patients with diabetes. Circulation 103: 2668-2673, 2001.

31. Dhalla NS, Pierce GN, Innes IR and Beamish RE: Pathogenesis of cardiac dysfunction in diabetes mellitus. Can J Cardiol 1: 263-281, 1985. 